\title{
A new RING for SUMO: wrestling transcriptional responses into nuclear bodies with PIAS family E3 SUMO ligases
}

\author{
Peter K. Jackson ${ }^{1}$ \\ Department of Pathology and Department of Microbiology and Immunology, Stanford University School of Medicine, \\ Stanford, California 94305-5324, USA
}

Ubiquitin is known for its catabolic role in directing protein degradation when added to proteins in polyubiquitin chains, causing the targeted proteins to bind to and be degraded by the $26 \mathrm{~S}$ proteasome. But when added as a monomer, ubiquitin can play an important role in noncatabolic processes ranging from histone regulation and endocytosis to virus budding (for review, see Hicke 2001). Recently a number of ubiquitin-like molecules (Ubls), including the protein SUMO (ㅁmall ubbiquitin-related modifier; also called sentrin), Nedd8/Rub1, and several even more exotic Ubls (Muller et al. 2001; Ohsumi 2001), have been found added to target proteins through isopeptide bonds to lysine side chains. Here, monoubiquitin and its Ubl cousins appear to function as posttranslational modifiers of protein function and likely reflect a more ancestral role for the ubiquitin structural fold in regulating protein activity. The biological roles of Ubl modification range even more broadly. Notably, modification by SUMO can play critical roles in both nuclear and cytoplasmic processes, including nuclear transport, the regulation of transcriptional processes, the targeting of transcriptional regulators to subnuclear domains, and the regulation of mitosis (Melchior and Hengst 2000; Hochstrasser 2001; Muller et al. 2001). Recently, an exciting new class of enzymes regulating the addition of SUMO to target proteins, called SUMO E3 ligases, has been described in yeast and mammals (Johnson and Gupta 2001; Kahyo et al. 2001; Takahashi et al. 2001). In this issue of Genes \& Development, Sachdev and colleagues from the Grosschedl laboratory report that a member of this family of proteins, called PIASy, regulates the activity and subnuclear localization of the Wnt-responsive transcription factor LEF1 by directing SUMO addition to LEF1 (Sachdev et al. 2001). The implication is that this family of SUMO modifying

${ }^{1}$ E-MAIL pjackson@cmgm.stanford.edu; FAX (650) 725-6902. Article and publication are at http://www.genesdev.org/cgi/doi/10.1101/ gad.955501. enzymes may regulate a variety of transcriptional-and nontranscriptional-responses by altering protein activity and localization.

\section{SUMO modification of target proteins: it's as easy as $\mathrm{E} 1, \mathrm{E} 2, \mathrm{E} 3$}

In vertebrates, SUMO is found in three forms: SUMO-1, SUMO-2, and SUMO-3 (SUMO-2 and SUMO-3 are very similar). SUMO-1 versus SUMO-2 and SUMO-3 appear to modify distinct targets. RanGAP, the GTPase-activating protein critical for controlling nuclear import, was the first identified SUMO-modified protein; here, SUMO modification appears to be important for RanGAP binding to the nuclear pore, although the biological importance of the modification remains less clear (Melchior and Hengst 2000).

In yeast, a single variant, Smt3, is essential for progression through mitosis. Although a variety of SUMOmodified species have been observed in yeast, the best characterized are several of the septins, a group of GTPbinding cytoskeletal proteins that form the bud neck in yeast and establish the site of cytokinesis (Johnson and Blobel 1999; Takahashi et al. 1999). The septins are modified by SUMO in early mitosis, suggesting a mitotic role for the modification. Indeed, yeast deficient in Smt3 or in the specific SUMO-modifying enzyme Ubc9 arrest in mitosis (Hochstrasser 2001). However, septin mutations deficient in SUMO modification do not show any discernable cell cycle phenotype (Johnson and Blobel 1999|. Therefore, neither the role of SUMO modification of septins nor the SUMO targets responsible for SUMO's role in mitosis are clear. Nonetheless, septins provide a convenient substrate to define the enzymes directing SUMO modification.

SUMO modification proceeds by a three-step enzyme shuttle analogous to ubiquitin addition (Melchior 2000). For ubiquitin, an ATP-dependent activation step couples ubiquitin by a thiolester bond to the E1, ubiquitin-acti- 
vating enzyme. In turn, ubiquitin is transferred to the reactive cysteine of one of several E2 ubiquitin-conjugating enzymes. Typically, an E3 ubiquitin ligase combines with the charged E2 to facilitate formation of an isopeptide bond between ubiquitin and the target protein (Jackson et al. 2000). E3s typically use protein-protein interaction domains to bind to and select specific targets and either a zinc-binding RING finger domain or a HECT domain to stimulate polyubiquitin chain formation. How ubiquitin is then assembled into a polyubiquitin chain remains unclear, but the chain is sufficient for binding to the $26 \mathrm{~S}$ proteasome and degradation, although other factors that assist in this process are becoming known (for review, see Pickart 2001).

In the case of SUMO, adenylation of SUMO and the formation of an E1-SUMO thiolester bond are accomplished by an E1 SUMO-activating enzyme similar to the E1 ubiquitin enzymes (Muller et al. 2001). An interesting evolutionary twist is that the $\mathrm{N}$-terminal adenylation function and the C-terminal thiolester binding site found in the ubiquitin E1 are assembled from two polypeptides in both the yeast and human variants of the SUMO E1. Following SUMO-E1 activation, SUMO is transferred to the E2 SUMO-conjugating enzyme Ubc9, which is homologous to the ubiquitin E2s. Additional enzymes for the processing of SUMO and the presence of SUMO-deconjugating enzymes (or isopeptidases) suggest that additional steps may be important for the regulation or dynamics of the sumoylation process (for review, see Muller et al. 2001).

Earlier biochemical studies showed that SUMO E1 and E2 were biochemically sufficient for efficient transfer of SUMO to specific target proteins (Melchior 2000). For SUMO modification, a consensus sequence $\Psi \mathrm{KxE}$ (where $\Psi$ is a hydrophobic residue) is required for SUMO modification both in vivo and in vitro using just E1 and E2 enzymes. Ubiquitination site selection appears to require merely the lysine side chain, and in many cases mutagenesis studies have failed to reveal preferred ubiquitination sites. The apparent addition of SUMO as a monomer led to a suspicion that the addition of monomeric Ubls might not require E3s. But this suspicion was erased by the recent discovery of factors that both resemble the RING finger domain found in many ubiquitin E3 ligases and serve a similar biochemical function for SUMO addition (for recent review, see Hochstrasser 2001). Even the observation that SUMO addition is monomeric appears now to be determined more by conditions in the cell, as these in vitro studies suggest that the addition of poly-SUMO chains is possible, at least in vitro.

Two groups used genetic interaction (two-hybrid) screens to identify SUMO-binding proteins. One study identified a human SUMO ligase that appears to be sufficient for SUMO modification of the p53 tumor suppressor (Kahyo et al. 2001), and the other identified a yeast SUMO ligase for septins (Takahashi et al. 2001). A comprehensive study from Johnson and Gupta (2001) studied the role of two highly related proteins, Sizlp and Siz2p. The authors show that Sizlp functions as an E3 SUMO ligase in vitro, requires the conserved zinc-binding cysteines within the variant RING finger domain for this activity, and demonstrated the requirements for Siz1 (and to a small degree Siz2) for sumoylation of both septins and the majority of yeast sumoylated species in vivo. A siz2 mutant has much more subtle deficiencies in SUMO modified species, although a siz1-siz2 double mutant does reduce the small residual sumoylation seen in a siz1 mutant. Surprisingly, even the siz1-siz2 double mutant did not show the mitotic arrest seen with smt3 or ubc9 mutants, suggesting that Smt3p and Ubc9p have broader activities. One possibility is that Siz1 and Siz2 are not the only E3 SUMO ligases. Another is that Siz1/ Siz2 functions can supercharge the sumoylation engine, but that SUMO ligases are not so critical for the basal level of sumoylation activity required for yeast mitosis. Indeed, because the SUMO modification of septins does not appear to be the essential role of Ubc9 or Smt3, the identity of the essential SUMO targets for mitosis in yeast remains to be discovered.

The in vitro results of Johnson and Gupta (2001) show that Sizl can enhance E1-E2-dependent sumoylation, but that at high concentrations of E1 and E2, the SUMO modification of septin substrates is independent of E3, possibly explaining why the E3 function is not strictly required in vivo. Even more surprising is that the Siz1 E3 could stimulate the formation of septin substrates with multiple SUMO additions. Addition of methylated SUMO, which blocks the autosumoylation by modifying the lysine side chains critical for SUMO-SUMO conjugates, inhibited what appears to be chain formation. The significance of poly-SUMO chains is not apparent, and the fact that they have not been clearly observed on Western blots of SUMO-modified proteins may only reflect the activity of a host of SUMO isopeptidases in cell lysates. However, if SUMO can form poly-SUMO chains in vitro, do they form in vivo, and what might their function be? Although proteasome targeting would be one possibility, studies discussed below show that SUMO can stabilize some proteins, which argues against proteasome involvement.

At this point, we also have a very limited understanding of how the sumoylation process itself is regulated within the cell. However, much like what happened with the recent molecular identification of the E3 ubiquitin ligases, the molecular identification of the SUMO ligases will certainly facilitate biochemical and cell biological studies. In the case of ubiquitination of target proteins, fairly elaborate requirements can restrict ubiquitination to specific locations and times in a cells life (for two illustrative examples, see Jaquenoud et al. 1998; Furstenthal et al. 2001).

There is currently no clear molecular basis for how SUMO addition regulates protein activity. One possible mechanism is that SUMO modification changes some property of the target protein, such as conformation or recruitment of binding partners. Notably, SUMO addition may provide a tether to specific SUMO-binding domains within protein complexes (discussed below), thereby directing protein targeting. Another mechanism 
is suggested by two recent studies showing that SUMO addition to lysines normally used for ubiquitylation can block ubiquitylation and proteolytic destruction of specific proteins. In one example, the $\mathrm{Mdm} 2$ ubiquitin ligase is sumoylated on a specific lysine to block its autoubiquitylation and self-annihilation (Buschmann et al. 2000). The model is that, in this state, the p53 tumor suppressor is ubiquitylated and kept unstable in resting cells. Here, the activation of de-sumoylation following DNA damage allows Mdm2 self-ubiquitylation and destruction, thereby stabilizing p53 to allow the initiation of cell cycle arrest and the activation of DNA repair. However, the mechanism by which de-sumoylation is triggered is not known, and there has been some controversy over the basic observations leading to the model (Melchior and Hengst 2000). In another example, SUMO addition to the transcriptional regulator I $\mathrm{B}$ B blocks its ubiquitylation and destruction by the $\mathrm{SCF}^{\beta-T r C P}$ complex (Desterro et al. 1998), thereby stabilizing the abil-

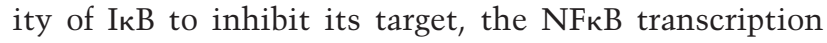
factor.

\section{The PIAS gene family: transcriptional regulators and more}

The Siz proteins share significant homology within the critical SUMO E3 ligase RING domain to the PIAS family of transcriptional regulators, suggesting functional conservation of the Siz/PIAS- or SP-RING E3 SUMO ligases (Hochstrasser 2001). In mammals, the PIAS proteins were first discovered as transcriptional coregulators of the JAK-STAT pathway (Liu et al. 1998). The binding of cytokines to cell surface receptors activates the Janus, or JAK, family of tyrosine kinases, which phosphorylate a family of at least seven cytoplasmic transcription factors termed STATs (signal transducer and activator of transcription). STATs mediate specific transcriptional responses. PIAS1 (protein inhibitor of activated STAT1) was identified as a specific inhibitor of STAT1 signaling, but conversely can enhance the transcriptional activity of nuclear hormone receptors (Liu et al. 1998; Kotaja et al. 2000; Gross et al. 2001). In vertebrates, four PIAS proteins (PIAS1, PIAS3, PIASx, and PIASy) are known (Liu et al. 1998). PIAS1 and PIAS3 inhibit DNA binding of the STAT1 and STAT3 factors (Chung et al. 1997; Liu et al. 1998). In contrast, the PIASy protein represses STAT1 and the activity of the androgen receptor without affecting DNA binding (Gross et al. 2001). In Drosophila, the PIAS orthologs dPIAS and stat92E cooperate to regulate eye and blood cell development, presumably by transcriptional regulation (Betz et al. 2001). However, an even more general role for PIAS family proteins was suggested by the characterization of the suppressor of position effect variegation, Su(var)2-10 (or ZIMP), as a Drosophila PIAS (Hari et al. 2001). Deficiencies in Su(var)2-10 caused strong effects on both mitotic chromosome condensation and the organization of interphase chromatin. Interestingly, the initial identification of yeast Sizl was through genetic interaction with the condensin complex (Strunnikov et al. 2001). Thus, the question of whether PIAS proteins function to regulate the specific activity of transcription factors or more generally regulate chromatin structures was raised. The paper from the Grosschedl group in this issue gives us the beginnings of an answer.

\section{Regulating transcriptional responses by SUMO: transcriptional activity and subcellular localization}

The interphase nucleus is organized into distinct domains, including the nuclear matrix and the chromatin structures of the chromosomes themselves. The nuclear matrix or interchromatin space contains several subnuclear domains. These include the promyelocytic leukemia (PML) nuclear bodies (also called PML oncogenic domains or PODs), which are dot-like structures (0.2-0.5 $\mu \mathrm{m}$ within the $\sim 12$ - $\mu \mathrm{m}$ nuclear sphere) found in most mammalian cells and first defined by the localization of the PML gene product, a transcriptional regulator originally identified as an oncogenic fusion protein associated with PML (Zhong et al. 2000). Nuclear bodies are somewhat heterogeneous and appear to regulate transcriptional responses both positively and negatively by organizing specific chromatin-associated regulators within them. The nuclear bodies are dynamic, changing in size, number, and composition during the cell cycle, and are strongly affected by physiological stresses and a variety of viral infections (see Muller et al. 2001). A central role for PML in the organization of nuclear bodies is suggested by the effect that perturbing PML has on these subnuclear domains. Notably, in $\mathrm{PML}^{-/-}$mice, nuclear bodies are lacking and cells lose growth control (Wang et al. 1998). PML can also interact with the transcriptional corepressor CBP to promote its localization to nuclear bodies (Zhong et al. 2000), interact with multiple corepressors and histone deacetylases, and can target transcriptional regulators to PML nuclear bodies to cause their inactivation. PML may not be the only central player in nuclear bodies regulated by sumoylation. Notably, sumoylation of the Sp100 transcriptional regulator may alter its interaction with heterochromatin protein 1 (HP1), possibly connecting the regulation of heterochromatin regions to nuclear bodies (Seeler et al. 1998; Muller et al. 2001).

The appearance and function of PML nuclear bodies appears to be strongly determined by the conjugation of PML with SUMO-1 (for review, see Hay 2001). Sumoylated PML is selectively localized to nuclear bodies, and mutants of PML lacking SUMO-modification sites fail to target to nuclear bodies, although they still remain associated with the insoluble nuclear matrix (Muller et al. 1998). Here the dynamics of SUMO addition and removal may be of considerable importance, and although specific SUMO ligases for PML and Sp100 have not been identified, the various PIAS proteins now become strong candidates.

In the study by the Grosschedl group, new regulators of the Wnt-signaling LEF1 transcription factor were sought by a two-hybrid screen, which identified a mem- 
ber of the PIAS family, PIASy (Sachdev et al. 2001). Broadly, their results show that PIASy is a strong candidate for the SUMO ligase important for controlling sumoylation and the ability of LEF1 to activate transcription. In addition, the ability of the PIASy SUMO ligase to regulate LEF1-dependent transcription reflects the association of PIASy with the nuclear matrix and the ability of PIASy to control nuclear body localization of a number of transcriptional regulators, including LEF1 itself.

PIASy interacts with LEF1 through its N-terminal SAP (SAF-A, Acinus, PIAS) domain, which is both necessary and sufficient for LEF1 binding. The SAP domain was defined by its sequence similarity to the nuclear matrixbinding protein SAF-A (Kipp et al. 2000), and, indeed, the authors find that this region of PIASy is necessary to bind to a matrix attachment region (MAR), but not a mutant MAR site, in an electrophoretic gel shift assay. It is not yet clear whether the SAP domain is also necessary for PIASy to target to nuclear bodies, although it appears likely.

Within LEF1 itself, PIASy appears to bind primarily, but not exclusively, to the C-terminal HMG domain, a well-studied DNA-binding motif, and another DNA interaction site for LEF1 (Giese et al. 1991). SAP domains are found in the other members of the PIAS family of transcription factors and may mediate PIAS interactions with distinct classes of target proteins and with sites on DNA or chromatin. Given the conservation of the SAP and RING domains, it will be important to carefully test and validate which specific PIAS family members regulate any SUMO-dependent physiological response and to be cautious as to whether overexpression of specific PIAS regulators maintains that specificity.

Coexpression of PIASy with variants of LEF1 caused a consistent $18-\mathrm{kD}$ shift, which Western blotting established as a SUMO-2/3-modified form (but not SUMO-1 or ubiquitin modified) of LEF1. Coexpression of LEF1 with SUMO-1 versus SUMO-2 suggested that SUMO-1 could be added to LEF1, but less efficiently. Because the C-terminal domain of PIASy resembled a RING finger, the zinc-binding region known to be important in ubiquitin ligases (Jackson et al. 2000; Hochstrasser 2001), the authors tested the effect of mutations in a conserved cysteine and a histidine within this potential RING motif. They found that these mutants were defective in the induction of PIASy-mediated sumoylation of LEF1 both in vivo and in vitro. Additionally, mutation of both $\Psi \mathrm{KxE}$ consensus sites in LEF1 caused a reduction in LEF1 sumoylation. Interestingly, mutations in the RING motif also caused a reduction in PIASy binding to LEF1, suggesting that although the SAP domain is necessary for this interaction, the RING motif does contribute to binding. Whether this contribution reflects a change in affinity or whether the RING motif stimulates an activity important for binding remains to be seen. Interestingly, overexpression of PIASy caused a substantial change in the total amount of SUMO-modified protein seen in extracts, suggesting PIASy may direct sumoylation of a sizeable fraction of the total SUMOmodified pool. This result underscores the importance of further validating the role of PIASy without overexpression.

What then does sumoylation do to LEF1? The authors examine several known biochemical properties of LEF1 and find that sumoylation does not alter the ability of LEF1 to bind DNA, nor the ability of LEF1 to associate with its Wnt-signaling coactivator $\beta$-catenin. However, overexpression of PIASy does repress LEF1-dependent transcriptional activation using a LEF1-binding-site transcriptional reporter, but does not affect the basal activity of the reporter. PIASy also represses $\beta$-catenin-stimulated transcription in the same assays, further supporting its role in the Wnt signaling pathway.

The authors further explore the generality of the repressive effect and find that PIASy can: (1) also repress transcription activity of the LEF1-related protein TCF1; (2) repress independent LEF1-containing control regions; and $(3)$ inhibit the activity of a reporter from the T-cell receptor $\alpha$ gene, which is stimulated by LEF1 in conjugation with the Ets1 and AML1 transcription factors. Indeed, other workers have found that PIASy can antagonize the activation of STAT1, p53, and the androgen receptor (AR) (Zhong et al. 2000). An interesting contrast is PIAS1, which can inhibit DNA binding of STAT1, and yet stimulate the transcriptional activity of AR (Kotaja et al. 2000; Zhong et al. 2000; Gross et al. 2001). Thus, with the caution that many of these conclusions are based on overexpression studies, the PIAS proteins can potentially both positively and negatively regulate transcription. It will be of interest to understand how PIAS sumoylation might mediate both activating and repressive responses (discussed below).

If SUMO modification of LEF1 inhibits its capacity for transcriptional activation, and yet LEF1 can still bind DNA or $\beta$-catenin, what does sumoylation physically do? The localization of endogenous and expressed PIASy to nuclear bodies suggested one possibility. Is PIASy mediating its effect in nuclear bodies? The authors tested whether overexpression of PIASy can affect targeting of LEF1 to nuclear bodies. Examination of a tagged LEF1 protein showed localization throughout the nucleus in transfected COS cells. Coexpression of PIASy strongly redistributed LEF1 to nuclear bodies, an effect enhanced by coexpression of SUMO-1 or SUMO-2. To test whether this effect required the SUMO ligase activity, the authors also transfected the RING mutant of PIASy. Not only did this RING mutant fail to target to nuclear bodies, but it was also unable to redistribute a mutant of LEF1, suggesting that the RING finger and likely the sumoylation activity of PIASy are important for both the localization of both PIASy and its targets to nuclear bodies. Moreover, this targeting strongly correlated with transcriptional repression by PIASy.

So which comes first, the targeting or the repression? It seems that PIASy requires its SAP targeting sequence for nuclear matrix association. For other proteins, targeting to the nuclear matrix is correlated with localization to nuclear bodies (Zhong et al. 2000). However, if the SUMO E3 activity is important for localizing both PIASy itself and LEF1 to nuclear bodies, it is possible that the 
sumoylation of several proteins, including other PIASy targets, serve to organize specific protein complexes onto nuclear matrix sites within nuclear bodies, possibly through receptor proteins containing SUMO binding sites. Although no clear example is known, the role of SUMO in targeting RanGAP to nuclear pores and PML to nuclear bodies would suggest the presence of SUMObinding proteins. A recent study suggests the presence of specific ubiquitin-binding domains within a number of different proteins, including proteasomal units (Hofmann and Falquet 2001). Given the extensive parallels between ubiquitin and SUMO, a search for SUMO-binding functions, possibly within the known SUMO regulators themselves, may be productive.

One central SUMO-modified organizer of nuclear bodies is PML itself, and it seems likely that one of the PIAS family members would stimulate PML sumoylation and its recruitment to nuclear bodies. The number of sumoylated factors in nuclear bodies and the valency and multimerization state of the known factors is unclear, but it is possible that complexes of sumoylated proteins and SUMO-binding proteins are organized within matrix- and chromatin-associated regions within these bodies.

The authors specifically examine whether the LEF1 mutant lacking the SUMO modification sites was localized to nuclear bodies when cotransfected with PIASy. Interestingly, the un-sumoylated mutant of LEF1 was still relocalized to nuclear bodies. Following the model suggested above, it could be that the SUMO modification of other nuclear body factors, like Sp100 or PML, recruit LEF1. LEF1 sumoylation, therefore, may assist but not be strictly required for its assembly into transcription repression complexes by PIASy.

The lack of a strict requirement for the sumoylation sites on the LEF1 target reflects a logic familiar to those of us who have studied regulation by protein phosphorylation. In many cases, a particular kinase mediates the phosphorylation state of a central regulator of some signaling pathway. Both the kinase and the regulator are essential for the pathway, and the phosphorylation of the regulator by the kinase perfectly correlates with the ability of the kinase to stimulate signaling. And yet detailed (and sometimes painful) mapping and mutagenesis of phosphorylation sites reveal no strict requirement for the phosphorylation (Furstenthal et al. 2001). In many cases, the answer proves to be that the kinase regulates several factors in the pathway and phosphorylation contributes globally to the regulation, but no single factor's phosphorylation is central. This is easiest to understand when several proteins form a complex and phosphorylation promotes or blocks a number of phosphorylationdependent protein-protein interactions.

One model, therefore, is that SUMO modification promotes protein-protein interactions and that these are critical for the assembly of a transcription repression complex (or, more broadly, a transcriptional regulation complex) at specific matrix-associated sites within the nucleus. These interactions could be attributable to the ability of SUMO to promote some other aspect of pro- tein-protein interaction (e.g., recruit chaperones) or to providing binding sites for SUMO-binding domains. Of considerable interest is the role of SUMO isopeptidases in this process. If SUMO ligases can indeed promote poly-SUMO chain formation and yet only SUMO monomer addition is readily observed, the isopeptidases would be predicted to be very active. If the transcriptional responses (and, indeed, many other regulatory events) require rapid activation and inactivation, it may be that the reversibility of SUMO addition and removal is critical for the dynamic assembly of regulatory complexes.

The evolutionary conservation of the enzymes regulating addition of ubiquitin and Ubls has been the subject of a recent review (Hochstrasser 2000). The striking conservation of the Ubls themselves and of the E1 and E2 enzymes, and the isopeptidases fits well with the model that a primordial ubiquitin-like molecule evolved to allow posttranslational modification of proteins by isopeptide bond formation. Given our model here, a critical feature of Ubl modification is the ability to reversibly add and remove Ubls. The recent suggestion of Ubl interaction motifs would provide one mechanism for Ubls to change protein function, by promoting reversible protein-protein interactions through domains like the UIM domain (Hofmann and Falquet 2001). Because some of the Ubl-interacting proteins may serve chaperone functions, the consequences of these binding events may modify not merely the association state of the Ubl-modified proteins, but possibly their conformation also. Once the enzymes that promote mono-Ubl addition and removal were established during evolution, specific Ubls like ubiquitin and SUMO could have evolved to allow addition of poly-Ubl chains. A poly-Ubl chain would minimally improve the avidity of modified proteins for Ubl-binding regions, but could further evolve to create specialized protein surfaces on the poly-Ubl chain, much as has been described for ubiquitin chains (Sloper-Mould et al. 2001). Additionally, poly-ubiquitin chains could also provide a means to increase the range over which modified proteins would bind their Ubl binding partners.

One of the more puzzling questions is how ubiquitylation reactions evolved to couple to the proteasome, which may predate the evolutionary appearance of ubiquitin. If poly-Ubl chains evolved to provide a way to couple to UIM and related domains, the presence of these chains provides a functional link between the ubiquitylated target protein and the UIM domains on the $19 S$ regulatory cap of the proteasome. One can imagine that the polyubiquitin chain was not originally present to restrict proteasome entry, but, instead to simply facilitate proteasome interaction. At some later time in evolution, the importance of regulating ubiquitin-dependent proteolysis may have provided a driving force to restrict proteasome entry only to ubiquitylated proteins.

The exciting discovery of the SUMO ligases and the puzzles posed by trying to understand their functional properties will undoubtedly stimulate many new bouts of SUMO wrestling. Notably, do other SUMO ligases 
exist, are there specific ligases for the various forms of SUMO, and do other Ubls require similar E3 ligases? Understanding how SUMO modification changes protein function will require detailed biochemical and structural approaches. Finally, identifying the substrates of specific SUMO ligases and defining how sumoylation alters the function of these substrates should be a winning technique (shitatenage) for getting a grip on a wide range of biological problems.

\section{References}

Betz, A., Lampen, N., Martinek, S., Young, M.W., and Darnell, Jr., J.E. 2001. A Drosophila PIAS homologue negatively regulates stat92E. Proc. Natl. Acad. Sci. 98: 9563-9568.

Buschmann, T., Fuchs, S.Y., Lee, C.G., Pan, Z.Q., and Ronai, Z. 2000. SUMO-1 modification of Mdm2 prevents its self-ubiquitination and increases $\mathrm{Mdm} 2$ ability to ubiquitinate p53. Cell 101: 753-762.

Chung, C.D., Liao, J., Liu, B., Rao, X., Jay, P., Berta, P., and Shuai, K. 1997. Specific inhibition of Stat3 signal transduction by PIAS3. Science 278: 1803-1805.

Desterro, J.M., Rodriguez, M.S., and Hay, R.T. 1998. SUMO-1 modification of $\mathrm{I} \kappa \mathrm{B} \alpha$ inhibits NF-кB activation. Mol. Cell 2: 233-239.

Furstenthal, L., Swanson, C., Kaiser, B.K., Eldridge, A.G., and Jackson, P.K. 2001. Triggering ubiquitination of a CDK inhibitor at origins of DNA replication. Nat. Cell Biol. 3: 715722 .

Giese, K., Amsterdam, A., and Grosschedl, R. 1991. DNA-binding properties of the HMG domain of the lymphoid-specific transcriptional regulator LEF-1. Genes \& Dev. 5: 2567-2578.

Gross, M., Liu, B., Tan, J., French, F.S., Carey, M., and Shuai, K. 2001. Distinct effects of PIAS proteins on androgen-mediated gene activation in prostate cancer cells. Oncogene 20: $3880-3887$.

Hari, K.L., Cook, K.R., and Karpen, G.H. 2001. The Drosophila $\mathrm{Su}($ var)2-10 locus regulates chromosome structure and function and encodes a member of the PIAS protein family. Genes \& Dev. 15: 1334-1348.

Hay, R.T. 2001. Protein modification by SUMO. Trends Biochem. Sci. 26: 332-333.

Hicke, L. 2001. Protein regulation by monoubiquitin. Nat. Rev. Mol. Cell Biol. 2: 195-201.

Hochstrasser, M. 2000. Evolution and function of ubiquitin-like protein-conjugation systems. Nat. Cell Biol. 2: E153-E157.

- 2001. SP-RING for SUMO: New functions bloom for a ubiquitin-like protein. Cell 107: 5-8.

Hofmann, K. and Falquet, L. 2001. A ubiquitin-interacting motif conserved in components of the proteasomal and lysosomal protein degradation systems. Trends Biochem. Sci. 26:347350.

Jackson, P.K., Eldridge, A.G., Freed, E., Furstenthal, L., Hsu, J.Y., Kaiser, B.K., and Reimann, J.D. 2000. The lore of the RINGs: Substrate recognition and catalysis by ubiquitin ligases. Trends Cell Biol. 10: 429-439.

Jaquenoud, M., Gulli, M.P., Peter, K., and Peter, M. 1998. The Cdc42p effector Gic $2 p$ is targeted for ubiquitin-dependent degradation by the SCFGrrl complex. EMBO I. 17: 53605373.

Johnson, E.S. and Blobel, G. 1999. Cell cycle-regulated attachment of the ubiquitin-related protein SUMO to the yeast septins. J. Cell Biol. 147: 981-994.

Johnson, E.S. and Gupta, A.A. 2001. An E3-like factor that pro- motes SUMO conjugation to the yeast septins. Cell 106: 735-744.

Kahyo, T., Nishida, T., and Yasuda, H. 2001. Involvement of PIAS1 in the sumoylation of tumor suppressor p53. Mol. Cell 8: 713-718.

Kipp, M., Gohring, F., Ostendorp, T., van Drunen, C.M., van Driel, R., Pryzybylski, M., and Fackelmeyer, F.O. 2000. SAFBox, a conserved protein domain that specifially recognizes scaffold attachment region DNA. Mol. Cell. Biol. 20: 74807489.

Kotaja, N., Aittomaki, S., Silvennoinen, O., Palvimo, J.J., and Janne, O.A. 2000. ARIP3 (androgen receptor-interacting protein 3) and other PIAS (protein inhibitor of activated STAT) proteins differ in their ability to modulate steroid receptordependent transcriptional activation. Mol. Endocrinol. 14: 1986-2000.

Liu, B., Liao, J., Rao, X., Kushner, S.A., Chung, C.D., Chang, D.D., and Shuai, K. 1998. Inhibition of Stat1-mediated gene activation by PIAS1. Proc. Nat1. Acad. Sci. 95: 10626-10631.

Melchior, F. 2000. SUMO: Nonclassical ubiquitin. Annu. Rev. Cell Dev. Biol. 16: 591-626.

Melchior, F. and Hengst, L. 2000. Mdm2-SUMO1: Is bigger better? Nat. Cell Biol. 2: E161-E163.

Muller, S., Matunis, M.J., and Dejean, A. 1998. Conjugation with the ubiquitin-related modifier SUMO-1 regulates the partitioning of PML within the nucleus. EMBO J. 17: 61-70.

Muller, S., Hoege, C., Pyrowolakis, G., and Jentsch, S. 2001. SUMO, ubiquitin's mysterious cousin. Nat. Rev. Mol. Cell Biol. 2: 202-210.

Ohsumi, Y. 2001. Molecular dissection of autophagy: Two ubiquitin-like systems. Nat. Rev. Mol. Cell Biol. 2: 211-216.

Pickart, C. 2001. Ubiquitin enters the new millenium. Mol. Cell 8: 499-504.

Sachdev, S., Bruhn, L., Sieber, H., Pichler, A., Melchior, F., and Grosschedl, R. 2001. PIASy, a nuclear matrix-associated SUMO E3 ligase, represses LEF1 activity by sequestration into nuclear bodies. Genes \& Dev. 15: (this issue).

Seeler, J.S., Marchio, A., Sitterlin, D., Transy, C., and Dejean, A. 1998. Interaction of SP100 with HP1 proteins: A link between the promyelocytic leukemia-associated nuclear bodies and the chromatin compartment. Proc. Natl. Acad. Sci. 95: 7316-7321.

Sloper-Mould, K.E., Jemc, J.C., Pickart, C.M., and Hicke, L. 2001. Distinct functional surface regions on ubiquitin. I. Biol. Chem. 276: 30483-30489.

Strunnikov, A.V., Aravind, L., and Koonin, E.V. 2001. Saccharomyces cerevisiae SMT4 encodes an evolutionarily conserved protease with a role in chromosome condensation regulation. Genetics 158: 95-107.

Takahashi, Y., Iwase, M., Konishi, M., Tanaka, M., Toh-e, A., and Kikuchi, Y. 1999. Smt3, a SUMO-1 homolog, is conjugated to $\mathrm{Cdc} 3$, a component of septin rings at the motherbud neck in budding yeast. Biochem. Biophys. Res. Commun. 259: 582-587.

Takahashi, Y., Toh-e, A., and Kikuchi, Y. 2001. A novel factor required for the SUMO1/Smt3 conjugation of yeast septins. Gene 275: 223-231.

Wang, Z.G., Ruggero, D., Ronchetti, S., Zhong, S., Gaboli, M., Rivi, R., and Pandolfi, P.P. 1998. PML is essential for multiple apoptotic pathways. Nat. Genet. 20: 266-272.

Zhong, S., Salomoni, P., and Pandolfi, P.P. 2000. The transcriptional role of PML and the nuclear body. Nat. Cell Biol. 2: E85-E90. 


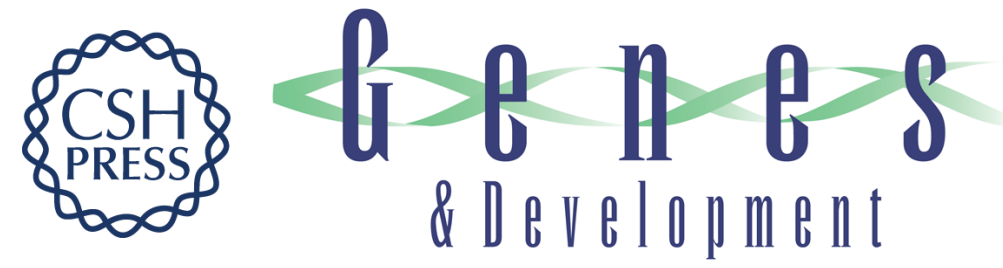

\section{A new RING for SUMO: wrestling transcriptional responses into nuclear bodies with PIAS family E3 SUMO ligases}

Peter K. Jackson

Genes Dev. 2001, 15:

Access the most recent version at doi:10.1101/gad.955501

References This article cites 34 articles, 12 of which can be accessed free at: http://genesdev.cshlp.org/content/15/23/3053.full.html\#ref-list-1

License

Email Alerting

Receive free email alerts when new articles cite this article - sign up in the box at the top Service right corner of the article or click here.

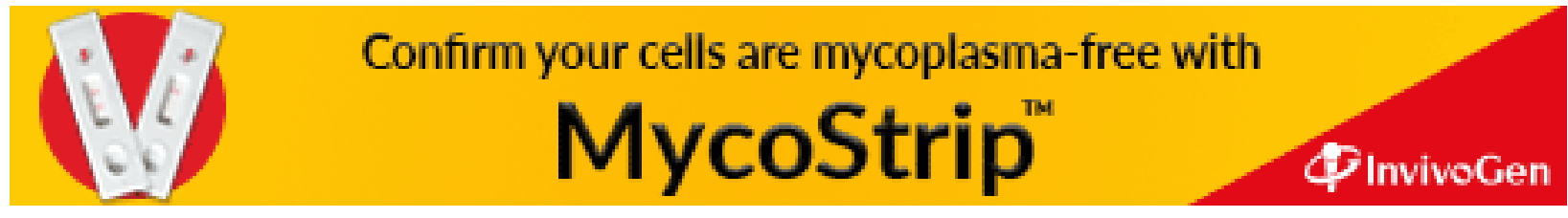

